\title{
Variedades clínicas de micosis fungoide en una cohorte
}

\author{
María Antonieta Domínguez-Gómez*, César A. Reyes-Salcedo, Martha A. Morales-Sánchez, \\ Gisela Navarrete-Franco, José Ramos-Garibay, Alberto González González-Maribet y \\ Cruz F. Jurado-Santa \\ Centro Dermatológico "Dr. Ladislao de la Pascua", Ciudad de México, México
}

\section{Resumen}

Introducción: La micosis fungoide es el linfoma primario de células $T$ en piel más frecuente, con expresividad clínica heterogénea. Objetivo: Reportar las variedades clínicas y las características sociodemográficas de pacientes con micosis fungoide tratados en un hospital dermatológico. Métodos: Se incluyeron 290 pacientes con diagnóstico clínico e histopatológico de micosis fungoide atendidos en el transcurso de 11 años. Se realizó descripción sociodemográfica de los pacientes, quienes se clasificaron conforme las variantes clínicas e histopatológicas. Resultados: $58 \%$ de los casos de micosis fungoide se presentó en mujeres y $42 \%$ en hombres. La variedad clínica más común fue la clásica en $46.2 \%$; la discrómica representó $35.2 \%$, del cual la hipopigmentada fue la más representativa (7.6 \%); la poiquilodérmica constituyó 4.1 \% y la foliculotrópica, $3.1 \%$. La variedad papular se presentó en seis pacientes $(2.1 \%)$, la de placa única en tres (1\%) y la ictiosiforme, siringotrópica y la piel laxa granulomatosa, en un paciente cada una. La variedad granulomatosa se encontró en 0.7 \% y $1.4 \%$ presentó eritrodermia. Conclusiones: La variedad clínica más frecuente de micosis fungoide fue la clásica en fase de placa, seguida de las variedades discrómicas. Otras variedades clínicas representaron $18.6 \%$.

PALABRAS CLAVE: Micosis fungoide. Linfoma cutáneo. Variedades clínicas. Cohorte retrospectiva.

\section{Clinical variants of mycosis fungoides in a cohort}

\section{Abstract}

Introduction: Mycosis fungoides (MF) is the most common primary skin T-cell lymphoma, which is characterized for a heterogeneous clinical expressivity. Objective: To report clinical variants and sociodemographic characteristics in patients with MF under the care of a dermatological hospital. Methods: 290 patients with MF clinical and histopathological diagnosis attended to over the course of 11 years were included. Sociodemographic description of patients was made, who were classified according to clinical and histopathological variants. Results: MF was recorded in $57.9 \%$ of women and $42 \%$ of men. The most common clinical variant was the classic type in $46.2 \%$; dyschromic variants accounted for $35.2 \%$, out of which hypopigmented MF was the most representative (17.6\%); poikilodermatous MF accounted for $4.1 \%$, and folliculotropic, for $3.1 \%$. The papular variant occurred in six patients $(2.1 \%)$, the single-plaque variety in three $(1 \%)$, and the ichthyosiform, syringotropic and granulomatous slack skin varieties occurred in one patient each. The granulomatous variant was found in $0.7 \%$, and 1.4 $\%$ had erythroderma. Conclusions: The most common MF clinical variant was classic plaque stage, followed by dyschromic variants. Other clinical variants accounted for $18.6 \%$.

KEY WORDS: Mycosis fungoides. Cutaneous lymphoma. Clinical variants. Retrospective cohort.

Correspondencia:

*María Antonieta Domínguez-Gómez

E-mail: dominguez035@yahoo.com.mx
Fecha de recepción: 10-02-2020

Fecha de aceptación: 29-05-2020

DOI: 10.24875/GMM.20000052
Gac Med Mex. 2021;157:43-49

Disponible en PubMed

www.gacetamedicademexico.com

0016-3813/@ 2020 Academia Nacional de Medicina de México, A.C. Publicado por Permanyer. Este es un artículo open access bajo la licencia CC BY-NC-ND (http://creativecommons.org/licenses/by-nc-nd/4.0/). 


\section{Introducción}

La micosis fungoide (MF) es el linfoma cutáneo de células $T$ más frecuente junto con el síndrome de Sezary; representa $50 \%$ de los casos y se caracteriza por proliferación de linfocitos $T$ atípicos con epidermotropismo. ${ }^{1}$ Maurelli et al. ${ }^{2}$ reportaron una incidencia anual de linfomas cutáneos en Europa de 10 por millón de habitantes; $71 \%$ correspondió a linfomas cutáneos de células T y de 50 a $73 \%$, a MF.

Los linfomas cutáneos primarios son un grupo heterogéneo de linfomas extraganglionares no Hodgkin con un tropismo particular hacia la piel; se dividen de acuerdo con su estirpe celular, se consideran de bajo potencial de malignidad y su pronóstico es diferente al de los linfomas sistémicos. Solo 1 a $2 \%$ de los casos puede presentar diseminación sistémica, estadio de la enfermedad en el que la supervivencia es pobre y el desenlace es fatal. ${ }^{3}$

La etiopatogenia de los linfomas cutáneos primarios sigue siendo estudiada, aunque la teoría de estimulación antigénica a largo plazo es la más reconocida. La respuesta inflamatoria ante estímulos persistentes provoca expansión clonal continua de células $T$ y la activación de vías dependientes de citocinas, lo que puede explicar inicialmente el infiltrado de linfocitos atípicos. También se han considerado las infecciones virales como desencadenantes, especialmente por virus de Epstein-Barr, variantes del parvovirus (B19, LaL1/K1,V9), herpes virus tipo 7 (HHV-7) y algunos poliomavirus humanos (HPyVs6, HPyVs7). ${ }^{4}$

La Organización Mundial de la Salud y la Organización Europea para la Investigación y Tratamiento del Cáncer reconocen la MF clásica, la piel laxa granulomatosa, la reticulosis pagetoide y la variedad foliculotrópica en el listado de clasificación de linfomas cutáneos. ${ }^{5}$

La evolución clásica, descrita por Alibert-Bazin, se caracteriza por la progresión desde una fase macular eritematosa a la aparición de placas infiltradas para terminar en neoformaciones en la fase tumoral. Sin embargo, existen numerosos reportes de casos de variedades clínicas no registradas en el listado anterior. ${ }^{6}$

Aunque la MF representa una entidad poco prevalente, es importante conocer las variantes de expresión clínica para la detección y tratamiento oportuno. Agar et al. ${ }^{7}$ realizaron el estudio más extenso, con 1502 pacientes de quienes describieron algunas características demográficas y el pronóstico de la enfermedad. Asimismo, Peña-Romero et al. ${ }^{8}$ reseñaron los hallazgos en una cohorte de pacientes con MF hipopigmentada en el Hospital General de México.

El objetivo del presente estudio fue identificar las características sociodemográficas de una cohorte de pacientes con diagnóstico de MF en un periodo de 11 años en un centro de referencia nacional para atención al paciente con problemas de piel.

\section{Métodos}

Se realizó un estudio de cohorte retrospectivo en pacientes con diagnóstico de MF del Centro Dermatológico "Dr. Ladislao de la Pascua". El protocolo de investigación fue revisado y aprobado por los comités institucionales de investigación y de ética en investigación. Se revisaron los expedientes electrónicos de los pacientes con diagnóstico de MF, clasificados con la clave CIE-10 C.84, del 1 de enero de 2007 al 31 de diciembre de 2018. Se excluyeron los expedientes en los que no se dispusiera del reporte histológico confirmatorio.

Los datos que se obtuvieron de cada expediente incluyeron características sociodemográficas, curso clínico de la MF, estado clínico del paciente al momento de la última cita médica y tiempo de respuesta al tratamiento. El estado clínico del paciente al término del seguimiento se clasificó como remisión, persistencia y progresión con base en el grado de infiltración cutánea y de superficie corporal afectada:

- Remisión, si en la última visita médica documentada en el expediente electrónico el médico tratante indicó que el paciente se encontraba sin lesiones compatibles con MF ni infiltración cutánea.

- Persistencia, si el estadio TNM era menor al inicial.

- Progresión, si el estadio TNM era mayor al inicial.

La superficie corporal afectada estaba documentada en el expediente electrónico con el registro fotográfico estandarizado del cuerpo completo con el que se cuenta en el centro donde se realizó la investigación. Se definió como tiempo de respuesta al tratamiento al tiempo transcurrido desde que el médico comunicó al paciente el resultado del estudio histológico y se inició el tratamiento (fecha de visita médica posterior al registro electrónico del resultado de la biopsia) y la fecha de la visita médica en la cual se registró la remisión de las lesiones de MF por parte del médico tratante y que se corroboró la suspensión del tratamiento. 
Para el análisis estadístico se utilizó el programa IBM SPSS versión 19. La descripción de las variables cualitativas se llevó a cabo mediante proporciones, mientras que las variables cuantitativas con distribución normal se describieron mediante medias y desviación estándar. Para la descripción del tiempo de respuesta al tratamiento se utilizó la mediana y el rango intercuartilar. El análisis de variables se realizó con la prueba de chi cuadrada, comparando los resultados.

\section{Resultados}

Se identificaron 335 expedientes clínicos electrónicos con diagnóstico de MF, de los cuales solo 290 contaban con resultado histológico confirmatorio; 168 correspondieron a mujeres (57.9\%) y 122, a hombres (42.1\%), con una relación de 1.3:1. La media de edad fue de 43 años, el paciente con menor edad tuvo cinco años y el de mayor edad, 90 años. El grupo de edad más frecuente fue el de 31 a 45 años, que representó $29 \%$ de la población, seguido por el de 46 a 60 años (23.8\%). La mediana de la enfermedad fue de seis años.

\section{Características clínicas}

La variedad clásica se presentó en 134 pacientes, de los cuales 128 (44.1\%) se encontraron en fase de placa (Figura 1), seis en fase de mancha (1.7\%) y uno en fase tumoral $(0.3 \%)$. Las discromías se presentaron en 102 pacientes (35.2) y se clasificaron en hipopigmentadas e hiperpigmentadas. La variedad hipopigmentada (Figura 2) fue la segunda más frecuente en nuestro estudio, afectó a 51 pacientes (17.6\%). En cuanto a las hiperpigmentadas (Figura 3), se categorizaron en las que se presentaron en pliegues $(7.9 \%)$ y las que se ubicaron en otros sitios $(9.7 \%)$. La variedad poiquilodérmica (Figura 4) fue la tercera variedad más frecuente: se observó en 12 pacientes (4.1\%). La variedad foliculotrópica (Figura 5) se registró en nueve pacientes (3.1\%), la papular en seis $(2.1 \%)$, la eritrodérmica en cuatro $(1.4 \%)$, la de placa única en tres (1\%), la granulomatosa en dos $(0.7 \%)$ y la piel laxa granulomatosa, siringotrópica e ictiosiforme en un paciente cada una. La distribución detallada por sexo se muestra en la Tabla 1.

En $5.2 \%$ de los pacientes se encontraron diferentes formas clínicas coexistentes, las cuales se catalogaron como polimorfas; la combinación más frecuente fue la variedad en placas con poiquilodermia, hiperpigmentación e hipopigmentación (Figura 6). En más de

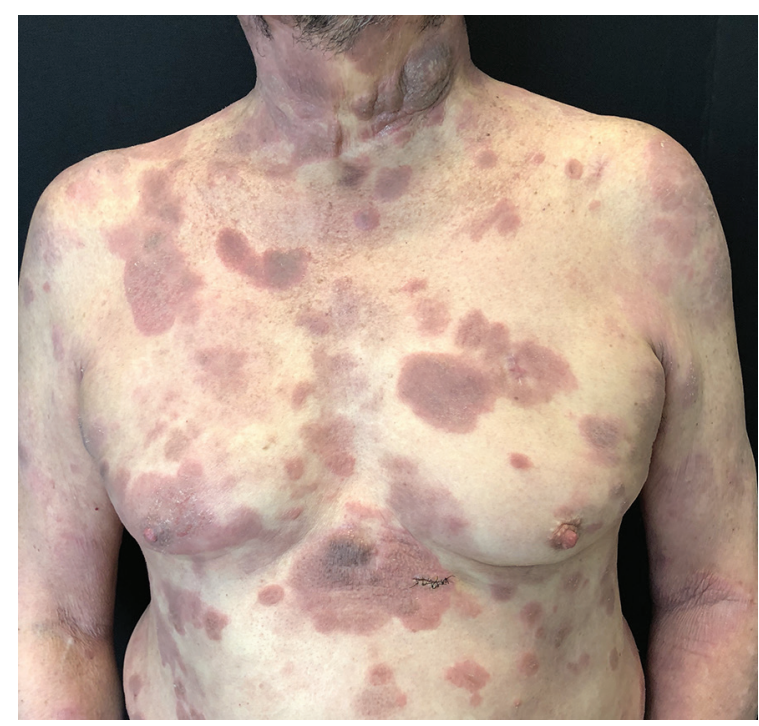

Figura 1. Paciente con micosis fungoide clásica en fase de placa.

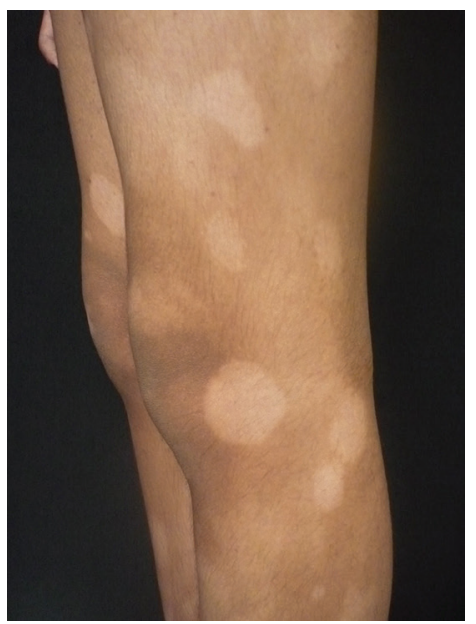

Figura 2. Paciente con micosis fungoide variedad hipopigmentada.

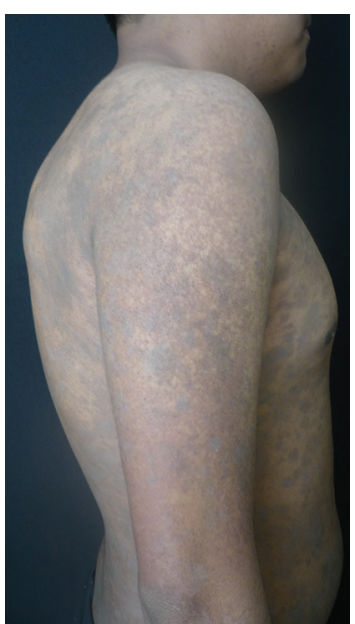

Figura 3. Paciente con micosis fungoide variedad hiperpigmentada. 


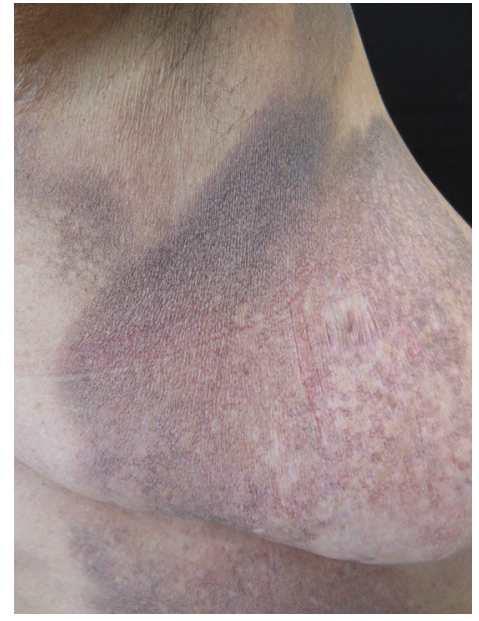

Figura 4. Paciente con micosis fungoide variedad poiquilodérmica.

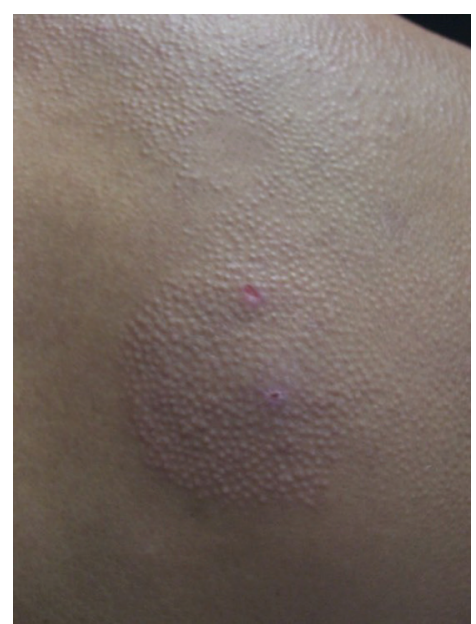

Figura 5. Paciente con micosis fungoide variedad foliculotrópica.

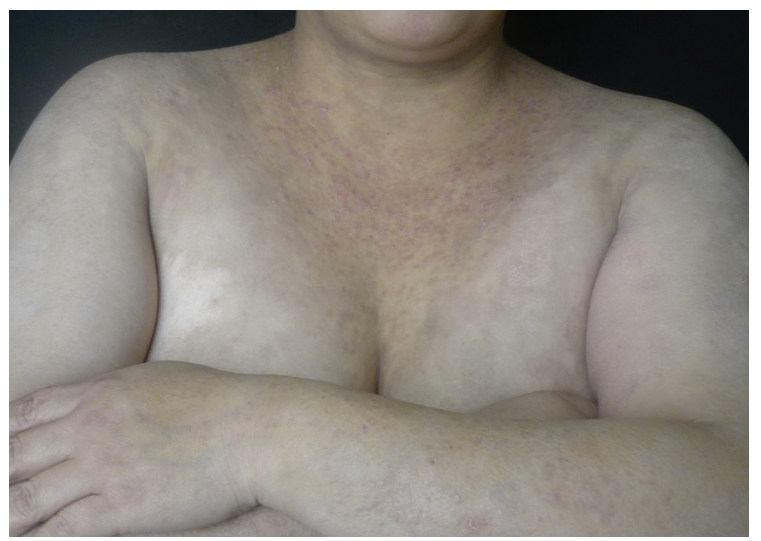

Figura 6. Paciente con micosis fungoide polimorfa con manchas hipopigmentadas, placas eritematoinflitradas, manchas hiperpigmentadas y pápulas eritematosas.
Tabla 1. Variedades clínicas de micosis fungoide en pacientes adultos y pediátricos

\begin{tabular}{|c|c|c|c|c|c|c|}
\hline \multirow[t]{2}{*}{ Tipo } & \multicolumn{2}{|c|}{ Adultos } & \multicolumn{2}{|c|}{ Niños } & \multirow{2}{*}{$\begin{array}{l}\% \text { del } \\
\text { total }\end{array}$} & \multirow[t]{2}{*}{$p$} \\
\hline & Sexo & $n$ & Sexo & $n$ & & \\
\hline \multirow{2}{*}{$\begin{array}{l}\text { Clásica: fase de } \\
\text { mancha }\end{array}$} & $\mathrm{F}$ & 2 & $\mathrm{~F}$ & 1 & \multirow[t]{2}{*}{1.7} & \multirow[t]{2}{*}{0.79} \\
\hline & $M$ & 1 & $\mathrm{M}$ & 1 & & \\
\hline \multirow{2}{*}{$\begin{array}{l}\text { Clásica: fase de } \\
\text { placa }\end{array}$} & $F$ & 63 & $\mathrm{~F}$ & 3 & \multirow[t]{2}{*}{44.1} & \multirow[t]{2}{*}{0.001} \\
\hline & $M$ & 56 & $\mathrm{M}$ & 5 & & \\
\hline \multirow{2}{*}{$\begin{array}{l}\text { Clásica: fase } \\
\text { tumoral }\end{array}$} & $\mathrm{F}$ & 0 & $\mathrm{~F}$ & 0 & \multirow[t]{2}{*}{0.3} & \multirow[t]{2}{*}{0.69} \\
\hline & $M$ & 1 & $\mathrm{M}$ & 0 & & \\
\hline \multirow[t]{2}{*}{ Hiperpigmentada } & $\mathrm{F}$ & 18 & $\mathrm{~F}$ & 1 & \multirow[t]{2}{*}{9.7} & \multirow[t]{2}{*}{0.47} \\
\hline & $M$ & 5 & $\mathrm{M}$ & 4 & & \\
\hline \multirow[t]{2}{*}{ Hipopigmentada } & $\mathrm{F}$ & 22 & $\mathrm{~F}$ & 10 & \multirow[t]{2}{*}{17.6} & \multirow[t]{2}{*}{$<0.001$} \\
\hline & $M$ & 10 & $\mathrm{M}$ & 9 & & \\
\hline \multirow[t]{2}{*}{ Foliculotrópica } & $\mathrm{F}$ & 3 & $\mathrm{~F}$ & 1 & \multirow[t]{2}{*}{3.1} & \multirow[t]{2}{*}{$<0.84$} \\
\hline & $\mathrm{M}$ & 5 & M & 0 & & \\
\hline \multirow[t]{2}{*}{ Siringotrópica } & $F$ & 1 & $\mathrm{~F}$ & 0 & \multirow[t]{2}{*}{0.3} & \multirow[t]{2}{*}{$<0.69$} \\
\hline & $\mathrm{M}$ & 0 & $\mathrm{M}$ & 0 & & \\
\hline \multirow[t]{2}{*}{ Pliegues } & $\mathrm{F}$ & 16 & $\mathrm{~F}$ & 0 & \multirow[t]{2}{*}{7.9} & $<0.18$ \\
\hline & $M$ & 6 & $M$ & 1 & & \\
\hline Granulomatosa & $\mathrm{F}$ & 1 & $\mathrm{~F}$ & 0 & 0.7 & $<0.58$ \\
\hline & $\mathrm{M}$ & 1 & $\mathrm{M}$ & 0 & & \\
\hline Piel laxa & $\mathrm{F}$ & 1 & $\mathrm{~F}$ & 0 & 0.3 & $<0.69$ \\
\hline granulomatosa & $M$ & 0 & $M$ & 0 & & \\
\hline Placa única & $F$ & 1 & $\mathrm{~F}$ & 0 & 1 & $<0.49$ \\
\hline & $\mathrm{M}$ & 2 & $\mathrm{M}$ & 0 & & \\
\hline Papular & $\mathrm{F}$ & 4 & $\mathrm{~F}$ & 2 & 2.1 & $<0.15$ \\
\hline & $M$ & 0 & $\mathrm{M}$ & 0 & & \\
\hline Poiquilodérmica & $\mathrm{F}$ & 12 & $\mathrm{~F}$ & 0 & 4.1 & $<0.16$ \\
\hline & $\mathrm{M}$ & 0 & $\mathrm{M}$ & 0 & & \\
\hline Polimorfa & $\mathrm{F}$ & 5 & $\mathrm{~F}$ & 0 & 5.2 & $<0.43$ \\
\hline & $M$ & 9 & $M$ & 1 & & \\
\hline Ictiosiforme & $\mathrm{F}$ & 1 & $\mathrm{~F}$ & 0 & 0.3 & $<0.69$ \\
\hline & $\mathrm{M}$ & 0 & M & 0 & & \\
\hline Eritrodérmica & $\mathrm{F}$ & 1 & $\mathrm{~F}$ & 0 & 1.4 & $<0.43$ \\
\hline & $\mathrm{M}$ & 3 & $\mathrm{M}$ & 0 & & \\
\hline Urticariforme & $\mathrm{F}$ & 1 & $\mathrm{~F}$ & 0 & 0.3 & $<0.69$ \\
\hline & $M$ & 0 & $\mathrm{M}$ & 0 & & \\
\hline
\end{tabular}


$50 \%$ de los pacientes, la micosis fungoide fue diseminada y los sitios más afectado fueron el tronco ( $86 \%)$ y las extremidades superiores e inferiores (77 y $74 \%$, respectivamente); el cuello se afectó en $29 \%$ de los pacientes y la cabeza en $23 \%$.

\section{Respuesta al tratamiento}

Las modalidades terapéutcias fueron manejo conservador, fototerapia, inhibidores de calcineurina, inmunosupresores, esteroides y retinoides tópicos o sistémicos, de acuerdo con el caso. De ellas, la fototerapia fue utilizada en 157 pacientes (54\%), de los cuales 109 (69.4\%) recibieron PUVA (psoraleno por vía oral y exposición posterior a radiación ultravioleta A) en cabina vertical y 48 (30.6\%) PUVA-sol. En 36 pacientes se utilizaron ambas modalidades. En relación con la respuesta al tratamiento, 219 presentaron remisión $(75.5 \%), 68(22.6 \%)$ remisión total y 151 (52\%), remisión parcial con disminución de las lesiones. Los 70 pacientes restantes presentaron persistencia de las lesiones, 52 dejaron de asistir por razones ajenas al tratamiento, incluyendo cambio de domicilio o de servicio médico, 10 pacientes continuaban en vigilancia y se encontraban en terapia con radiación ultravioleta $A$ en cabina vertical y seis fueron derivados a tercer nivel de atención.

\section{Discusión}

La expresividad clínica de la micosis fungoide es heterogénea; en nuestra población, el comportamiento en adultos fue distinto al de los pacientes pediátricos. La variedad clásica en fase de placa se presentó más en los adultos que en los niños, en quienes la variedad hipopigmentada fue la más frecuente ( $p=0.001$ y $p<0.001$, respectivamente). A diferencia de $60 \%$ de la población de Reino Unido reportada por Agar et al., $44 \%$ de la población presentó la variedad clásica en fase de placa. La variedad hipopigmentada se observó en $17 \%$ de la población, en la misma proporción encontrada por Peña Romero et al., quienes realizaron un reporte en pacientes en el Hospital General de México. En la literatura, la incidencia es mayor en hombres con una edad media de 50 años, con un índice comparativo de 2:1 respecto a las mujeres. ${ }^{9}$ En nuestro estudio, las mujeres fueron las más afectadas (57.9\%), con un índice comparativo con los hombres de 1.3:1. Atribuimos las diferencias clínicas a las características de la población mexicana, en la cual los fototipos son mayores que en los caucásicos.

La micosis fungoide puede imitar clínica e histopatológicamente diversas dermatosis de pronóstico variable, por lo que emitir el díagnóstico representa un reto para el dermatólogo y dermatopatólogo. Skov et $a ._{.10}^{10}$ realizaron un estudio retrospectivo en el Hospital de Copenhague, donde estudiaron el retraso del diagnóstico de micosis fungoide; reportaron la revisión de 157 pacientes con diagnóstico histopatológico de micosis fungoide, de los cuales $25 \%$ fue catalogado desde la primera biopsia y la media de tiempo para emitir el diagnóstico fue de 2.3 años. En nuestro centro, la media fue de cuatro años.

Diversas teorías pretenden explicar la pérdida de pigmento. En 1982, Breathnach et al.11 postularon al infiltrado linfocitario como el desencadenante de la degeneración de melanocitos con subsecuente alteración en la melanogénesis como una respuesta al proceso inflamatorio. Goldberg et al. ${ }^{12}$ demostraron que no existían cambios en los melanocitos y sugirieron que el defecto se encontraba en la transferencia de melanosomas. Flaxman et al. ${ }^{13}$ observaron disminución en el receptor de tirosina cinasa CD117, por lo que postularon la teoría del efecto citotóxico de los linfocitos TCD8 Th-1, que resulta en inestabilidad y disfunción del receptor con pobre respuesta a factores de transcripción..$^{14}$

La variedad hiperpigmentada se observó en $17.6 \%$ de la población estudiada. Se dispone de pocos reportes de casos, el de Boulos et al. incluye la población más grande: $:^{15}$ una cohorte de 34 pacientes estadounidenses, en la cual $29 \%$ tuvo esta variedad, caracterizada por manchas hiperpigmentadas sin poiquilodermia, más frecuente en fototipos altos y en sujetos menores de 35 años.

Se desconoce el mecanismo por el cual se presenta la hiperpigmentación, algunas de las teorías que pretenden explicarla son la degeneración hidrópica de la capa basal con formación de melanófagos, el efecto citotóxico e inmunosupresor de los linfocitos TCD8 sobre los queratinocitos y las células basales. En 2016, Kondo et al. reportaron el incremento en el número de mastocitos con trasformación a células grandes, lo cual puede desencadenar mayor secreción de melanina. ${ }^{16}$

Las lesiones poiquilodérmicas se han considerado como una variedad clínica de la micosis fungoide clásica en fase de placa. Clínicamente se observan pápulas eritematosas confluentes con patrón reticular que semejan al liquen rojo plano y que típicamente 
afectan pliegues y tronco; la presencia de lesiones poiquilodérmicas en áreas fotoexpuestas debe hacer sospechar micosis fungoide, hasta no comprobar lo contrario. ${ }^{15}$ Se describe un patrón de pigmentación moteado que alterna con áreas de hipo e hiperpigmentación, atrofia y telangiectasias que recuerdan el papel para cigarro. Se refiere que son asintomáticas y que se presentan con más frecuencia en hombres de 40 a 44 años, aunque en nuestro estudio $100 \%$ de los casos fue del sexo femenino. ${ }^{17}$

La variedad foliculotropa es una de las variedades clínicas más reportadas en la literatura. Clínicamente se caracteriza por pápulas foliculares agrupadas que forman placas induradas asociadas a alopecia y prurito, el cual representan un parámetro de actividad de la enfermedad y que preferentemente afecta cara y cuello. ${ }^{18}$ Los hallazgos histopatológicos son células del infiltrado con disposición folicular, donde se visualiza degeneración mucinosa del folículo piloso. Se presenta habitualmente en varones, si bien se han descrito algunos casos en niños y adolescentes. ${ }^{19}$ Kovačič reportó a la micosis fungoide foliculotrópica como la variante más frecuente en adultos, a pesar de solamente abarcar el $10 \%$ de los casos. ${ }^{20}$

La respuesta a tratamiento fue favorable en $75 \%$ de los pacientes con mejoría total o parcial. La modalidad terapéutica más utilizada fue la fototerapia en cabina vertical con radiación ultravioleta $A$, considerada de primera línea de tratamiento de la micosis fungoide. ${ }^{21-23}$

Consideramos una limitante del estudio la pérdida de datos derivada de la falta de información en los expedientes clínicos, aunque el seguimiento estrecho en la clínica de fototerapia y el registro iconográfico aportaron información relevante.

En conclusión, nuestro estudio presenta una cohorte de pacientes mexicanos y proporciona un panorama acerca de las variedades clínicas de la MF; será conveniente realizar estudios para conocer la frecuencia de presentación, el curso clínico y el pronóstico de la enfermedad.

\section{Conflicto de intereses}

Los autores declaran no tener ningún conflicto de interés.

\section{Financiamiento}

La presente investigación no recibió ninguna beca específica de agencias de los sectores público, comercial, o sin ánimo de lucro.

\section{Responsabilidades éticas}

Protección de personas y animales. Los autores declaran que para esta investigación no se realizaron experimentos en seres humanos ni en animales.

Confidencialidad de los datos. Los autores declaran que siguieron los protocolos de su centro de trabajo sobre la publicación de datos de pacientes.

Derecho a la privacidad y consentimiento informado. Los autores obtuvieron el consentimiento informado de los pacientes o sujetos referidos en el artículo. Este documento obra en poder del autor de correspondencia.

\section{Bibliografía}

1. Peñate $Y$, Servitje $O$, Machan $S$, Fernández-de Misa R, Estrach MT, Ramón MD, et al. Registro de linfomas cutáneos primarios de la AEDV: primer año de funcionamiento. Actas Dermosifiliogr. 2018;109:610-616.

2. Maurelli M, Tessari G, Colato C, Schena D, Girolomoni G. Incidence and ten-year follow-up of primary cutaneous lymphomas: a single-centre cohort study. Eur J Dermatol. 2018;28(1):44-49.

3. Stadler R, Stranzenbach R. Molecular pathogenesis of cutaneous lymphomas. Exp Dermatol. 2018;27:1078-1083.

4. Bergallo M, Daprà V, Fava $P$, Ponti R, Calvi C, Montanari P, Fierro MT. DNA from human poliomaviruses, MWPyV, HPyV6, HPyV7, DPyV9 and HPyV12 in cutaneous T-cell lymphomas. Anticancer Res. 2018;38:4111-4114.

5. Willemze R, Jaffe ES, Burg G, Cerroni L, Berti E, Swerdlow SH, et al. WHO-EORTC classification for cutaneous lymphomas. Blood. 2016;105:3768-3785.

6. Muñoz-González H, Molina-Ruiz AM, Requena L. Variantes clínico-patológicas de micosis fungoide. Actas Dermatosifiliogr. 2016;108:192-208.

7. Agar NS, Wedgeworth E, Crichton S, Mitchell TJ, Cox M, Ferreira S, Whittaker SJ. Survival outcomes and prognostic factors in mycosis fungoides/sézary syndrome: validation of the revised International Society for Cutaneous Lymphomas/European Organisation for Research and Treatment of Cancer staging proposal. J Clin Oncol. 2010;28:4730-4739.

8. Peña-Romero AG, Montes-de Oca G, Fierro-Arias L, Arellano-Mendozal,Peniche-Castellanos A, Mercadillo-Pérez P. Micosis fungoide hipopigmentada: diferencias clínico-histopatológicas con respecto a la micosis fungoide en placas. Dermatol Rev Mex. 2016;60:387-396.

9. Cerroni L. Mycosis fungoides clinical and histopathologic features, differential diagnosis, and treatment. Semin Cutan Med Surg. 2018;37:2-10.

10. Skov AG, Gniadecki R. Delay in the histopathologic diagnosis of mycosis fungoides. Acta Derm Venereol. 2015;95:472-475.

11. Breathnach SM, McKee PH, Smith NP. Hypopigmented mycosis fungoides: report of five cases with ultrastructural observations. Br J Dermatol. 1982;1016:643-649.

12. Goldberg DJ, Schinella RS, Kechijian P. Hypopigmented mycosis fungoides. Specutlations about the mechanism of hypopigmentation. Am J Dermatopathol. 1986;8:326-330.

13. Flaxman BA, Sosis AC, van Scott EJ. Changes in melanosome distribution in Caucasoid skin following topical application of nitrogen mustard. J Invest Dermatol. 1973;60:321-326.

14. Bisherwal K, Singal A, Pandhi D, Sharma S. Hypopigmented mycosis fungoides: clinical, histological, and immunohistochemical remission induced by narrow-band ultraviolet B. Indian J Dermatol. 2017;52:203-206.

15. Boulous S, Vaid R, Aladily TN, Ivan DS, Talpur R, Duvic M. Clinical presentation, immunopathology, and treatment of juvenile-onset mycosis fungoides: a case series of 34 patients. J Am Acad Dermatol. 2014;71:1117-1126.

16. Kondo M, Igawa K, Munetsugu T, Shibama S, Nishizawa A, Tokoro S, et al. Increasing numbers of mast cells in skin lesions of hyperpigmented mycosis fungoides with large-cell transformation. Ann Dermatol. 2016;28:115-116.

17. Pankratov O, Gradova S, Tarasevich S, Pankratov V. Poikilodermatous mycosis fungoides: clinical and histopathological analysis of a case and literature review. Acta Dermatovenerol Alp Pannonica Adriat. 2015;24: 37-41.

18. Abbot RA, Sahni D, Robson A, Agar N, Whittaker S, Scarisbrick JJ Poikilodermatous mycosis fungoides; a study of its clinicopathological immunophenotypic and prognostic features. J Am Acad Dermatol. 2011;65:313. 
19. Robson A, Assaf C, Bagot M, Burg G, Calonje E, Castillo C, et al. Aggressive epidermotropic cutaneous CD8+ lymphoma: a cutaneous lymphoma with distinct clinical and pathological features. Report of an EORTC Cutaneous Lymphoma Task Force Workshop. Histopathology. 2015:67(4):425-441.

20. Boix-Vilanova, J, Corral-Magaña O, Martin-Santiago A, Escalas J. Folliculotropic mycosis fungoides in a pediatric patient with response to psoralen-ultraviolet A phototherapy. Photodermatol Photoimmunol Photomed. 2019;35:54-56.
21. Kovačič S. Folliculotropic mycosis fungoides: clinicopathological features. Acta Medico-Biotechnica 2018;11:11-17.

22. Leuchte K, Schlaak M, Stadler R, Theurich S, von Bergwelt-Baildon M. Innovative treatment concepts for cutaneous T-cell lymphoma based on microenvironment modulation. Oncol Res Treat. 2017;40:262-269.

23. Trautinger F, Eder J, Assaf C et al. European Organisation for Research and Treatment of Cancer consensus recommendations for the treatment of mycosis fungoides/Sezary syndrome - update 2017. Eur J Cancer. 2017;77:57-74. 